\title{
Rapid detection of chlorophyll content and distribution in citrus orchards based on low-altitude remote sensing and bio-sensors
}

\author{
Kejian Wang, Wentao Li, Lie Deng, Qiang Lyu, Yongqiang Zheng, Shilai Yi, \\ Rangjin Xie, Yanyan Ma, Shaolan $\mathrm{He}^{*}$ \\ (Citrus Research Institute of Chinese Academy of Agricultural Sciences, Southwest University, Beibei, Chongqing 400712, China)
}

\begin{abstract}
The accuracy of detecting the chlorophyll content in the canopy and leaves of citrus plants based on sensors with different scales and prediction models was investigated for the establishment of an easy and highly-efficient real-time nutrition diagnosis technology in citrus orchards. The fluorescent values of leaves and canopy based on the Multiplex 3.6 sensor, canopy hyperspectral reflectance data based on the FieldSpec4 radiometer and spectral reflectance based on low-altitude multispectral remote sensing were collected from leaves of Shatang mandarin and then analyzed. Additionally, the associations of the leaf SPAD (soil and plant analyzer development) value with the ratio vegetation index (RVI) and normalized differential vegetation index (NDVI) were analyzed. The leaf SPAD value predictive model was established by means of univariate and multiple linear regressions and the partial least squares method. Variable distribution maps of the relative canopy chlorophyll content based on spectral reflectance in the orchard were automatically created. The results showed that the correlations of the SPAD values obtained from the Multiplex 3.6 sensor, FieldSpec4 radiometer and low-altitude multispectral remote sensing were highly significant. The measures of goodness of fit of the predictive models were $R^{2}=0.7063, R M S E C V=3.7892, R E=5.96 \%$, and $R M S E P=3.7760$ based on $\mathrm{RVI}_{(570 / 800)}$ and $R^{2}=0.7343, R M S E C V=3.6535$, $R E=5.49 \%$, and $R M S E P=3.3578$ based on $\operatorname{NDVI}_{[(570,800)(570,950)(700,840)]}$. The technique to create spatial distribution maps of the relative canopy chlorophyll content in the orchard was established based on sensor information that directly reflected the chlorophyll content of the plants in different parts of the orchard, which in turn provides evidence for implementation of orchard productivity evaluation and precision in fertilization management.
\end{abstract}

Keywords: citrus, remote sensing, bio-sensor, chlorophyll detection, spectrum, ratio vegetation index (RVI), normalized differential vegetation index (NDVI), spatial distribution map

DOI: $10.25165 /$ j.ijabe.20181102.3189

Citation: Wang K J, Li W T, Deng L, Lyu Q, Zheng Y Q, Yi S L, et al. Rapid detection of chlorophyll content and distribution in citrus orchards based on low-altitude remote sensing and bio-sensors. Int J Agric \& Biol Eng, 2018; 11(2): 164-169.

\section{Introduction}

The chlorophyll content of citrus canopy leaves and plants is directly associated with the level of photosynthetic capacity and carbohydrate synthesis of the plants and forms a basic vital condition and nutritional support for high quality tree yields ${ }^{[1,2]}$. The timely diagnosis and investigation of chlorophyll content and its spatial distribution in a citrus orchard are important and necessary for nutrition diagnosis, production capacity evaluation and scientific fertilization decisions. The routine diagnostic method used for measuring chlorophyll content is extraction liquid

Received date: 2017-01-09 Accepted date: 2017-10-16

Biographies: Kejian Wang, Master, research interest: fruit information technology. Email: 992557031@qq.com; Wentao Li, Master, research interest: fruit information technology, Email: 1303726379@qq.com; Lie Deng, Professor, research interest: citrus information technology and precision agriculture. Email: denglie@cric.cn; Qiang Lyu, PhD, Associate Professor, research interest: precision agriculture. Email: lvqiang@cric.cn; Yongqiang Zheng, PhD, Associate Professor, research interest: fruit tree physiology. Email: zhengyongqiang@cric.cn; Shilai Yi, Associate Professor, research interest: citrus nutrition and agricultural information technology. Email: yishilai@cric.cn; Rangjin Xie, PhD, Associate Professor, research interest: fruit tree physiology. Email: xierangjin@cric.cn; Yanyan Ma, PhD, research interest: fruit tree physiology. Email: 445199776@qq.com.

*Corresponding author: Shaolan He, Associate Professor, research interest: citrus physiological and agricultural information technology. Citrus Research Institute, Southwest University, Beibei, Chongqing 400712, China. Tel: +8623-68349706, Email: heshaolan@cric.cn. colorimetric analysis. However, the measurement process is time-consuming, costly and, to some extent, destructive. Additionally, it is difficult to actualize the real-time and dynamic analysis of the change in chlorophyll content in large areas and to obtain the chlorophyll distribution in a tree canopy or individual plants. Efficiently, easy and accurate acquisition of the chlorophyll content information of different individual plants, or of plants in different parts of a citrus orchard, and understanding the chlorophyll content level and spatial distribution in an entire orchard in time are important for chlorophyll rapid diagnosis and precise plant fertilization in fruit trees. Compared with the conventional chemical chlorophyll content determination technology, the commercial application of the rapid monitoring for leaf SPAD (soil and plant analyzer development) values enables easier and more efficient acquisition of chlorophyll content. Moreover, with the availability of practical sensors and general techniques, it has become easy to understand the photosynthesis ability of the plants and evaluate the nutritional level of fruit trees ${ }^{[3]}$.

Many detection machines and technology research for measuring the chlorophyll content in leaves have been reported, including ultraviolet spectrophotometry, the fluorescence analysis method, the in vivo chlorophyll meter method, photoacoustic spectroscopy, high-performance liquid chromatography, and others ${ }^{[4]}$. The SPAD502 chlorophyll meter has been widely used for measuring and evaluating the relative chlorophyll content of leaves ${ }^{[5]}$. Markwell et al. ${ }^{[7,8]}$ described the SPAD chlorophyll meter as a reliable way to rapidly detect chlorophyll content in 
different cultivars of soybean, maize, sorghum and Arabidopsis thaliana, and in different genotypes of the same species of soybean; the correlation between SPAD values and chlorophyll content of both sides of the same sample were recorded. $\mathrm{Li}$ et al. ${ }^{[9]}$ found that SPAD values could be used to evaluate the status of nitrogen nutrition, which is highly correlated with leaf chlorophyll content, and to guide the production and use of fertilizers. Asai et al. ${ }^{[10]}$ used SPAD values to evaluate the relative chlorophyll content by studying the correlation between the application of biological carbon and the chlorophyll content. Pinar et al. ${ }^{[11]}$ and Gitelson et al. ${ }^{[12]}$ found that the red edge position could better reflect the canopy chlorophyll. Wang and $\mathrm{Ji}^{[13]}$ and Zhang et al. ${ }^{[14]}$ studied the fast detection method of chlorophyll content in wheat and rice based on hyperspectral and multispectral imaging methods. Deng et al. ${ }^{[15]}$ established a spectral pretreatment method for detecting the chlorophyll content in apple leaves based on the first order difference followed by wavelet packet denoising. In recent years, the FieldSpec4 series spectroradiometer from ASD Inc. in the U.S. has drawn great attention because of its high-resolution and full spectral coverage $(350-2500 \mathrm{~nm})$. The application of spectral reflectivity has made different progress in agriculture, forestry, agrology and other fields. FieldSpec 4 has certain advantages in terms of chlorophyll content detection at different spatial scales. The Mini-MCA12 multispectral array camera from Tetracam Inc. in the U.S. was produced based on the characteristic wavelength of agronomic traits and could obtain low-altitude remote sensing multispectral information of the plant canopy from unmanned aerial vehicles. Spectral information at the orchard scale further helps in the analysis and exploration of different physiological statuses of plants. The Multiplex 3.6 sensor from Force-A in France can obtain real-time canopy fluorescence information by the excitation of a light source and can be used in the evaluation of canopy color and nitrogen nutrition status. The real-time monitoring technology of chlorophyll content of a citrus canopy or an entire orchard helps in guiding the scientific management of fertilization. The chlorophyll content of citrus leaves based on low altitude remote sensing and canopy hyperspectral information can be used for the rapid prediction of individual nutritional levels and fruit-bearing potentials; a chlorophyll content distribution map can show the distribution differences in orchard nutrition and provide the basis for precision fertilization variable partition. Both will facilitate fine nutrition management and fertilizer savings. However, there are few reports of research in this area.

The values measured by the SPAD-502 chlorophyll meter were used in this study as the fundamental data of relative chlorophyll content. Comparisons were performed between the values measured by the SPAD-502 chlorophyll meter and several sensors such as the Multiplex 3.6 fluorescence spectrometer, the ASD FieldSpec4 and the Mini-MCA multispectral low-altitude remote sensing system. Additionally, the predictive feasibility of the predictive models for the SPAD values of leaf and canopy was analyzed. This study also explored a method for generating visibility maps of the spatial distribution of canopy chlorophyll content, which provides a new reference for the precise management of fruits or orchard nutrition.

\section{Materials and methods}

\subsection{Materials}

A citrus orchard $\left(25.4846^{\circ} \mathrm{N}\right.$ and $\left.110.2764^{\circ} \mathrm{E}\right)$ located in Huangbai village of Lingchuan County, Guilin city of the Guangxi Zhuang Autonomous Region was used as the experimental material.
A total of 128 8-year-old Shatang Mandarin plants (Citrus reticulata Blanco $c v$. shatangju) in different parts of the orchard were randomly selected for this study.

\subsection{Methods}

Collection of low-altitude remote sensing multispectral information: from 10:00 am to 15:00 am on sunny days, an unmanned aerial vehicle loaded with a Mini-MCA12 multispectral camera was used to collect the multispectral remote sensing image in the test area. The camera was produced by the Tetracam Company in the USA and contained 11 CMOS sensors with a pixel size of $1.3 \mathrm{M}(1280 \times 1024)$ and a filter with a diameter of $25 \mathrm{~mm}$. Additionally, a light sensor channel was armed for automatic correction of the errors that resulted from the change in light during remote sensing monitoring. The center wavelengths of the spectrum acquisition channel were as follows: $490 \mathrm{~nm}, 550 \mathrm{~nm}$, $570 \mathrm{~nm}, 671 \mathrm{~nm}, 680 \mathrm{~nm}, 700 \mathrm{~nm}, 720 \mathrm{~nm}, 800 \mathrm{~nm}, 840 \mathrm{~nm}$, $900 \mathrm{~nm}$ and $950 \mathrm{~nm}$. The half-wave bandwidth was $10 \mathrm{~nm}$ at $1^{\text {st }}-9^{\text {th }}$ bands, $20 \mathrm{~nm}$ at $10^{\text {th }}$ bands, $40 \mathrm{~nm}$ at $11^{\text {th }}$ bands. To ensure that the camera was always perpendicular to the ground, the M8 unmanned aerial vehicle was equipped with an orthographic pan-and-tilt, and the flight height of the unmanned aerial vehicle was $100 \mathrm{~m}$ with a flying speed about $6 \mathrm{~m} / \mathrm{s}$. It took the stationary point shooting and set up a stationary point for $50 \mathrm{~m}$, and two pictures were taken at each station. In this experiment, two pictures were spliced together, and then analyzed. The exposure time was $1 \mathrm{~ms}$, with triggered shooting. Four pieces of $3 \mathrm{~m} \times 3 \mathrm{~m}$ reflective cloth were deployed. The calibrated reflectance values of the cloth pieces were $3 \%, 12 \%, 34 \%$ and $56 \%$. The cloth pieces were laid near the experimentation area. To avoid interference with each other, the reflective cloth pieces were separated by more than $0.5 \mathrm{~m}$. The low-altitude remote sensing spectral reflectance is represented by RF in this paper.

Collection of visible-near infrared hyperspectral information in the canopy: Hyperspectral reflectance of the canopy was measured by the FieldSpec 4 spectroradiometer (FieldSpec 4 Standard-Res, ASD Inc., USA). The wavelength range of the FieldSpec4 ranged from $350-2500 \mathrm{~nm}$, the spectral resolutions were $3 \mathrm{~nm}$ at 350 $700 \mathrm{~nm}$ and $10 \mathrm{~nm}$ at $1400-2100 \mathrm{~nm}$, the sampling intervals were $1.4 \mathrm{~nm}$ at $350-1025 \mathrm{~nm}$ and $2 \mathrm{~nm}$ at $1025-2500 \mathrm{~nm}$, the scan time was $100 \mathrm{~ms}$, the number of channels was 2151 , and the field of view was $25^{\circ}$. The optical fiber probe was focused on the canopy and was $15-20 \mathrm{~cm}$ away from the canopy. Mirror reflections were avoided during the collection. The inclination angle of the optical fiber probe was kept constant as much as possible and the exposure time was $1 \mathrm{~ms}$. The measurement was repeated three times and the results were averaged. The white board was rectified every 15 min or when the light intensity was changed. The canopy spectral reflectance is represented by ASD.

Collection of UV-visible fluorescence information in the canopy: the fluorescence values of the canopy test plant were collected by the portable UV-visible fluorescence spectrometer (Multiplex 3.6) with the power of light source selected as cfg3 (cfg3 represents a power level). The detection probe of Multiplex 3.6 was focused on the middle of the canopy and $15-20 \mathrm{~cm}$ away from the canopy. Mirror reflection was avoided and parallel light was prevented from entering the sampling hole during the collection. Values at three different points were collected for each plant. The results were averaged as the Multiplex 3.6 canopy fluorescence value of the plant and are represented by 'outdoor'. The geographic coordinates and elevation information of each measurement point were synchronously recorded by the 
instrument's built-in GPS positioning system. A geographical distribution map of the orchard plants was drawn using Surfer 11 software. Multiplex 3.6 is composed of four excitation sources and three detection channels. The red light excited simple fluorescence ratio SFR_R was regarded as the relative content of chlorophyll $^{[17]}$.

Collection of simple leaf UV-visible fluorescence information: the leaf blade samples of test plants were collected from all directions in the middle and upper parts of the periphery. Fifteen leaves were collected from each plant, stored in an icebox and immediately brought back to the laboratory. The fluorescence value of a simple leaf was detected one-by-one by the Multiplex 3.6 sensor. The test leaf fully covered the sampling hole during collection. The measurements were repeated three times. The results were averaged and are represented by 'indoor'. Then, SPAD values of leaves were detected one-by-one by the SPAD502 and averaged as the relative content of chlorophyll.

\subsection{Data processing and modeling}

Firstly, the relationships between the SPAD values and different independent variables were analyzed. Then, the variables with higher correlation coefficient were selected for regression analysis ${ }^{[18]}$. Variables with better correlations were chosen to establish the partial least squares (PLS) prediction model, and the goodness-of-fit prediction models were tested. The independent variables used in this study including: simple leaf fluorescence value (indoor), canopy fluorescence value (outdoor), canopy hyperspectral reflectance (ASD), low-altitude remote sensing multispectral reflectance (RF), conversion processing LOG $(1 / R F)$, ratio of vegetation index (RVI) and normalized difference vegetation index (NDVI). Correlation analysis between single variables such as indoor and outdoor and the SPAD value were performed, and the significance of the results was determined. A univariate linear regression PLS model was sequentially performed to establish the correlation and the goodness-of-fit model was analyzed if the correlation was significant. For multi-variables, such as ASD, RF, LOG (1/RF), RVI and NDVI, the effective variables were extracted and then unitary or multiple linear regression analysis was performed to establish the prediction model for the number of variables.

The spectral reflectance data were computed using Excel 2016, and the fluorescent value was directly derived. The canopy hyperspectral reflectance was extracted by the ViewSpecPro software (ASD Inc., Longmont, Colorado, USA). The channel information of the low-altitude remote sensing image was combined and output by PixelWrench2, and the reflectivity was extracted from the output image by ENVI 4.7. Correlation analysis between all the independent variables and the SPAD values were firstly performed by SPSS 19.0. The independent variables with better correlations were then chosen to establish the model. The GUI_SPA K-S classification was performed at a modeling set/prediction set ratio of 3:1 by Matlab 2010b. Finally, the PLS prediction model was established by Unscrambler v9.7. The coefficient of determination $\left(R^{2}\right)$, average relative error (RE) and root mean square error (RMSE) were used to determine the goodness of fit for the models ${ }^{[19]}$.

The PLS modeling method is the most extensive and effective method currently used in the quantitative analysis of near infrared spectroscopy ${ }^{[20,21]}$. It is capable of effectively reducing the dimension and has characteristics such as improved reliability and data accuracy with high computational speed. To concisely and effectively compare the applicability of different detection methods, the PLS model was used to analyze and predict for all independent variables in this study.

The Kennard-Stone $(K-S)$ classification method was used for grouping. The samples with high spectrum disparities were selected into the calibration set, and other similar samples were selected into the validation set. In this way, all representative samples were selected into the calibration set and the scale and uniformity of the model could be increased ${ }^{[23]}$.

\subsection{Spatial distribution map of the relative content of chlorophyll in the canopy}

The region of interest (ROI) was selected from the low-altitude remote sensing image. The predictive content and the coordinate information of chlorophyll were obtained. The chlorophyll space distribution map was drawn by Surfer 11. The SPAD value and predictive value of Multiplex 3.6 were substituted to coordinate the information of the remote sensing image for drawing.

\section{Results and analysis}

\subsection{SPAD value of citrus leaves predicted by the Multiplex 3.6 sensor}

Correlation analysis was performed between the citrus simple leaf fluorescence average value in each plant measured by Multiplex 3.6 and the average SPAD value. The results showed that the correlation coefficient was significant at the 0.01 level.

The 128 fluorescence samples were divided into a modeling set with 96 samples and a prediction set with 32 samples. The results of the establishment and the prediction of the PLS forecasting model were shown in Table 1, line 2. The results showed that the model coefficient of determination $R^{2}$ was 0.5733 and the $R M S E C V$ was 4.5217 . The average relative error of the prediction set $R E$ was $9.03 \%$, and the RMSE was 5.4803 .

\subsection{SPAD value of the citrus canopy predicted by the Multiplex 3.6 sensor}

Correlation analysis was performed between the canopy fluorescence value of the plant measured by Multiplex 3.6 and the average leaf SPAD value. The results showed that the correlation coefficient was 0.546 , which was significant at the 0.01 level. The correlation coefficient between the SPAD value and the fluorescence value of the citrus canopy reached $0.546^{* *}$ and its PLS model were shown in Table 1 (line 2).

3.3 SPAD value of the citrus canopy predicted by FieldSpec 4

A total of 2151 bands of canopy visible and near-infrared reflectance were collected, and 1001 bands in the region of $350-1350 \mathrm{~nm}$ closely related to the agronomic characters were selected $^{[22][22]}$. The first-order derivative of the reflectance was calculated and is represented by DR. The relationships between DR and SPAD values in 1001 bands were analyzed and the characteristic wavelengths for modeling were screened. The results showed that the correlation coefficients $(r)$ were -0.529 , -625 and -311 at the three wave humps/valleys of DR at $452 \mathrm{~nm}$, $491 \mathrm{~nm}$ and $1263 \mathrm{~nm}$, respectively. The SPAD prediction model of chlorophyll relative content based on DRF, the DRF491 spectral reflectance were shown in Table 1 (lines 3 and 4, respectively).

\subsection{Canopy SPAD value prediction based on the low-altitude} multispectral information of the citrus orchard

Spectral image information obtained aerially by the Mini-MCA multispectral array camera was converted to the TIF image format after geometric correction and light intensity correction by software of the PixelWrench2 x64. The spectral information of each of the three channels was integrated to a TIF image and ENVI 4.7 was used to extract the brightness values of the corresponding 
wavelengths. The canopy SPAD predictive model was investigated by using RF, LOG(1/RF), RVI and NDVI as the independent variables. Among them, LOG (1/RF) indicated that the denary logarithm of the reciprocal of reflectance RVI was $R F_{X} / R F_{Y}$, NDVI was $\left(R F_{X}-R F_{Y}\right) /\left(R F_{X}+R F_{Y}\right)$, and $X, Y$ indicated the arbitrary bands.

SPAD value prediction study results showed that the relationship between RF and SPAD values was significant at the 0.01 level. The $r$ was greater than 0.7 at wavelengths of $490 \mathrm{~nm}$, $671 \mathrm{~nm}$ and $680 \mathrm{~nm}$. The correlation coefficient reached a maximum value of $r=-0.756$ at a wavelength of $680 \mathrm{~nm}$. The predictive model was established and tested by the method described in Section 3.1. The results were shown in Table 1, lines 5 and 6 , respectively.

SPAD value prediction study based on LOG (1/RF): Correlation analysis was performed between Canopy spectral reflectance and SPAD value for 128 plants. The results showed that the $r$ of all were greater than 0.7 between the SPAD value and the spectral reflectance at wavelengths of $490 \mathrm{~nm}, 671 \mathrm{~nm}$ and $680 \mathrm{~nm}$. The correlation coefficient reached a maximum value of $r=-0.735$ at a wavelength of $680 \mathrm{~nm}$. The results were shown in Table 1 , lines 7 and 8 , respectively.

SPAD value prediction study based on RVI: a total of 55 RVIs were obtained by the pairwise combination of each of the 11 spectral bands, and correlation analysis was performed between each RVI and the SPAD. The results showed that 53 RVI were significant at the 0.01 level. Among them, the correlation coefficients of 12 combinations, including $700 \mathrm{~nm} / 720 \mathrm{~nm}$,
$570 \mathrm{~nm} / 800 \mathrm{~nm}, 680 \mathrm{~nm} / 800 \mathrm{~nm}, 700 \mathrm{~nm} / 800 \mathrm{~nm}, 570 \mathrm{~nm} / 840 \mathrm{~nm}$, $720 \mathrm{~nm} / 840 \mathrm{~nm}, 570 \mathrm{~nm} / 900 \mathrm{~nm}, 700 \mathrm{~nm} / 900 \mathrm{~nm}, 570 \mathrm{~nm} / 950 \mathrm{~nm}$, $680 \mathrm{~nm} / 950 \mathrm{~nm}, 700 \mathrm{~nm} / 840 \mathrm{~nm}$ and $700 \mathrm{~nm} / 950 \mathrm{~nm}$, were greater than 0.8 and reached the maximum value of $r=-0.850$ at a wavelength of $570 \mathrm{~nm} / 800 \mathrm{~nm}$.

$\mathrm{RVI}_{570 \mathrm{~nm} / 800 \mathrm{~nm}}$ was selected to establish the univariate linear regression SPAD predictive model. Four combinations of RVI with correlation coefficients of $|r| \geq 0.830$, including $570 \mathrm{~nm} / 800 \mathrm{~nm}$, $570 \mathrm{~nm} / 950 \mathrm{~nm}, 700 \mathrm{~nm} / 800 \mathrm{~nm}$ and $700 \mathrm{~nm} / 950 \mathrm{~nm}$, were chosen to establish the multiple linear regression SPAD predictive model. The results were shown in Table 1, lines 9 and 10, respectively. SPAD value prediction study based on NDVI: a total of 55 NDVI were obtained by pairwise combination of each of the 11 spectral bands, and the correlation analysis between each NDVI and the SPAD were performed. The results showed that 53 NDVI were significant at the 0.01 level. Among them, the correlation coefficients of 11 combinations, including $(570 \mathrm{~nm}, 800 \mathrm{~nm})$, $(671 \mathrm{~nm}, 800 \mathrm{~nm}),(680 \mathrm{~nm}, 800 \mathrm{~nm}),(700 \mathrm{~nm}, 800 \mathrm{~nm}),(570 \mathrm{~nm}$, $840 \mathrm{~nm}),(720 \mathrm{~nm}, 840 \mathrm{~nm}),(570 \mathrm{~nm}, 900 \mathrm{~nm}),(700 \mathrm{~nm}, 900 \mathrm{~nm})$, $(570 \mathrm{~nm}, 950 \mathrm{~nm}),(680 \mathrm{~nm}, 950 \mathrm{~nm})$ and $(700 \mathrm{~nm}, 950 \mathrm{~nm})$, were greater than 0.8 and reached the maximum value of $(-0.849)$ at the

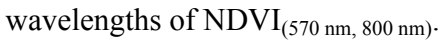

$\operatorname{NDVI}_{(570 \mathrm{~nm}, 800 \mathrm{~nm})}$ was selected to establish the univariate linear regression predictive model of the canopy SPAD. Three combinations of NDVI with a correlation coefficient of $r>0.830$ were chosen to establish the multiple linear regression predictive model of the canopy SPAD. The results were shown in Table 1, lines 11 and 12, respectively.

Table 1 SPAD predictive model with different input variables

\begin{tabular}{|c|c|c|c|c|c|c|}
\hline \multirow{2}{*}{$\begin{array}{c}\text { Serial } \\
\text { number }\end{array}$} & \multirow{2}{*}{ Independent variable } & \multirow{2}{*}{ Regression equation } & \multicolumn{2}{|c|}{ Modeling set } & \multicolumn{2}{|c|}{ Predictive set } \\
\hline & & & $R^{2}$ & RMSECV & $R E$ & RMSEP \\
\hline 1 & Indoor & $Y=33.75+32.6 X$ & 0.5733 & 4.5217 & $9.03 \%$ & 5.4803 \\
\hline 2 & Outdoor & $Y=14.1+18.49 X$ & 0.2914 & 6.2724 & $8.33 \%$ & 5.0766 \\
\hline 3 & $\mathrm{ASD}_{-\mathrm{DRF}_{491}}$ & $Y=62.56-89717 X$ & 0.3838 & 5.8006 & $7.38 \%$ & 4.9679 \\
\hline 4 & $\operatorname{ASD}^{-D R F}(X 1: 452, X 2: 491, X 3: 1263)$ & $Y=61.3-28954 X_{1}-59661 X_{2}-16816 X_{3}$ & 0.4657 & 5.4778 & $6.52 \%$ & 4.3988 \\
\hline 6 & $\mathrm{RF}_{(X 1: 490, X 2: 671, X 3: 680)}$ & $Y=68.33+537.2 X_{1}-621.9 X_{2}-488.9 X_{3}$ & 0.5484 & 4.7389 & $8.12 \%$ & 4.5339 \\
\hline 7 & $\operatorname{LOG}\left(1 / \mathrm{RF}_{680}\right)$ & $Y=7.047+26.98 X$ & 0.5235 & 5.0580 & $7.47 \%$ & 4.3210 \\
\hline 8 & $\operatorname{LOG}(1 / \mathrm{RF})_{(X 1: 490, X 2: 671, X 3: 680)}$ & $Y=15.15+8.298 X_{1}+7.961 X_{2}+6.876 X_{3}$ & 0.5114 & 5.1254 & $7.79 \%$ & 4.4784 \\
\hline 9 & $\mathrm{RVI}_{570 / 800}$ & $Y=75.92-160.7 X$ & 0.7063 & 3.7892 & $5.96 \%$ & 3.7760 \\
\hline 10 & $\operatorname{RVI}_{(X 1: 570 / 800, X 2: 570 / 950, X 3: 700 / 840, X 4: 700 / 900)}$ & $Y=78.46-37.22 X_{1}-36.29 X_{2}-44.2 X_{3}-41.58 X_{4}$ & 0.7562 & 3.4183 & $6.10 \%$ & 3.7537 \\
\hline 11 & $\operatorname{NDVI}_{(570,800)}$ & $Y=-24.04-102.6 X$ & 0.6995 & 3.8691 & $5.88 \%$ & 3.5239 \\
\hline
\end{tabular}

\subsection{Mapping of the SPAD value difference distribution of the} citrus orchard

Based on the modeling and prediction models and the integrated analysis of various indicators, we found that both RVI and NDVI predictive models showed good predictive effect on the canopy chlorophyll content. Considering the goodness of fit, the

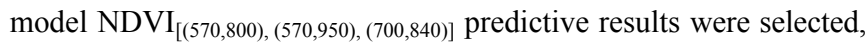
and the spatial distribution maps of the measured SPAD chlorophyll content in the orchard (Figure 1) and the $\operatorname{NDVI}_{[(570,800) \text {, }}$ (570,950), (700,840)] chlorophyll relative content (Figure 2) were generated by Surfer 11 according to the location information of the test plants. The chlorophyll content level and spatial distribution in different regions of the orchard and each plant were intuitively and clearly reflected by the spatial distribution map of the chlorophyll content. As seen in Figures 1 and 2, the trend of the highest and lowest points of the predictive values were the same as

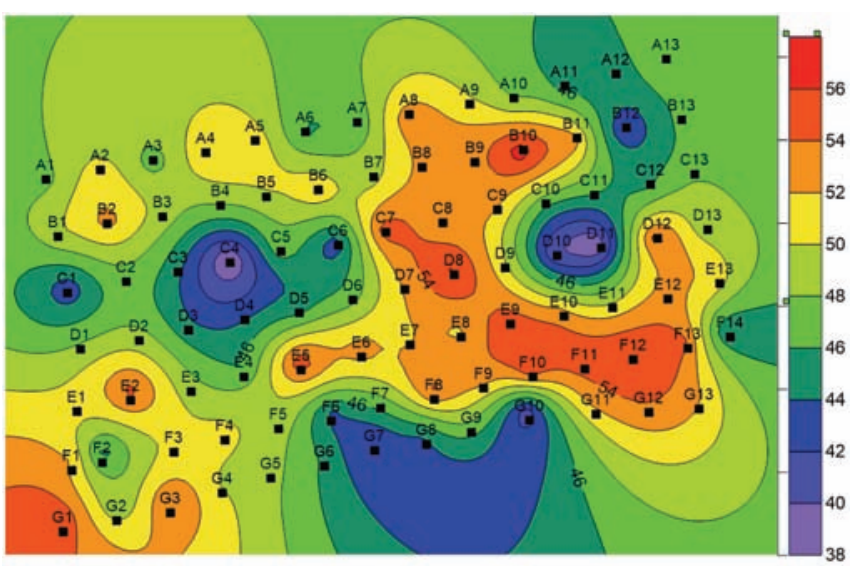

Figure 1 Spatial distribution of the measured SPAD chlorophyll relative content 


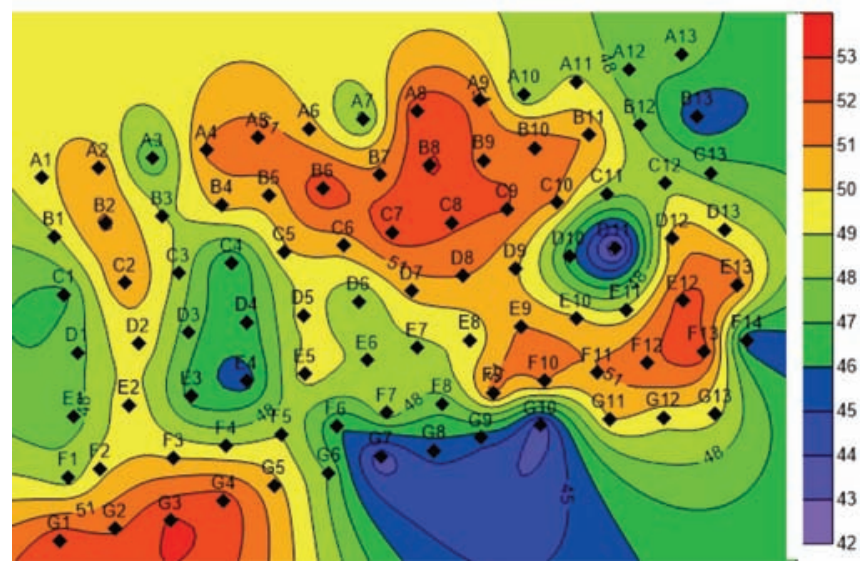

Figure 2 Distribution of chlorophyll relative content based on $\operatorname{NDVI}_{[(570,800),(570,950),(700,840)]}$

the measured value of SPAD, which indicated the feasibility of remote sensing monitoring of the canopy chlorophyll relative content in the orchard. The predictive models showed good predictive effect for canopy chlorophyll content.

\section{Discussion}

Netto et al. ${ }^{[6]}$, Markwell et al. ${ }^{[8]}$ and Uddling et al. ${ }^{[24]}$ found that the SPAD value was not completely and linearly related to the chlorophyll content by chemical detection; rather, the tiny differences between the SPAD value and chlorophyll content of different cultivars and leaf sides were within an acceptable range. The SPAD chlorophyll metric had high specificity and has been widely used as a reliable method for rapid nondestructive detection of chlorophyll. However, the actual measuring area of the SPAD chlorophyll metric was $2 \mathrm{~mm} \times 3 \mathrm{~mm}$. Taking the uneven chlorophyll distribution into account, it was necessary to measure several points on the same leaf to obtain a reliable value. This method is mainly applicable in diagnosing the chlorophyll content of single leaves. The Multiplex 3.6 fluorescence spectrometer has a light source, is not restricted to weather conditions and can also measure the fluorescence values of single leaves and the canopy. In addition to chlorophyll, it can also detect the content of leaf nitrogen nutrition ${ }^{[17]}$, anthocyanin, polyphenols and other substances. The data can be directly used and do not require transformation processing. The portability of the Multiplex 3.6 spectrometer is better than that of FieldSpec 4 but less than the SPAD chlorophyll meter. To maintain the fluorescent value within 20-4300, the light source power requires adjustment and the fluorescent values with different power thus cannot be compared. The measurement indices of the Multiplex 3.6 spectrometer are relatively specific and suitable for detecting the chlorophyll content of a canopy and individual leaves. FieldSpec 4 has rich spectral information and can be used in many types of composition detection but can easily be influenced by light. A slight change of light between the two whiteboard corrections may produce differences in results. The obtained spectral information needs to be processed by pretreatment to better reflect the chlorophyll content and the portability is thus poor. It is applicable for the comprehensive comparison of chlorophyll content and other material contents in the plant canopy; obtaining chlorophyll content and other mineral elements at the same time guides the diagnosis of nutrient diseases and insect pests. The Mini-MCA12 multispectral array camera contains most of the characteristic bands with good correlation with plant components, and reduces the workload of spectral processing. The spectral information can be obtained from the orchard scale, and the sampling error is reduced. However, skilled operation of the unmanned aerial vehicles is required. The method can be affected by weather conditions. It is suitable for the overall detection of chlorophyll content in the orchard and mineral nutrition level assessment. Therefore, different detection methods can be selected based on different needs.

The prediction results of this test were compared and it was found that the mean REs of the predicted and measured values for all prediction models of independent variable construction were within $10 \%$, with a minimum of $5.49 \%$. Among the models, the prediction results of forecast models that were built by the variables RVI and NDVI were the best. The $R^{2}$ of the RVI multiple regression model was up to 0.7562 . It was shown that the multiple regression analysis method could produce a higher prediction coefficient of determination with an increase in the input variables, but average relative errors of the predicted results did not decrease. This could be due to the over-fitting of multiple regression analysis ${ }^{[6]}$. Therefore, more independent variables will not be necessary to improve the goodness of fit of the model ${ }^{[24]}$. When establishing the linear regression model, the choice of input variables affects the predictive ability of the model. Additionally, different pretreatment methods and combined approaches of the spectrum affect the predictive ability of the model ${ }^{[27]}$; these are current research topics in experimental treatments. The extraction of the characteristic wavelength in FieldSpec 4 canopy hyperspectral reflectance was analyzed. It was found that the extraction effect was based on the PLS regression coefficient method, which was not as good as the extraction effect by analyzing the correlation of each band and selecting the wavelength with maximum correlation.

Therefore, the coefficients of determination in this method were all below 0.8 . The prediction effects were slightly lower than those of the single scale hyperspectral method ${ }^{[28]}$, which may be because of the influences of representative sampling, surface sediment, vertical direction and other factors ${ }^{[29]}$.

\section{Conclusions}

The spectral information of single leaves and the canopy acquired by the Multiplex 3.6 sensor well reflected the SPAD value of relative chlorophyll content of citrus leaves and the tree canopy. Under the conditions of this experiment, the correlation of single leaf fluorescence value and the SPAD testing value was better than the canopy fluorescence value.

For the spectral reflectance of the canopy based on the FieldSpec 4 sensor, the first derivation of reflectance at a wavelength of $491 \mathrm{~nm}$ showed maximum correlation with the canopy chlorophyll relative content in the citrus, with $r=-0.625$, which was a high significance. The established linear regression prediction model showed good predictive effect for canopy SPAD value.

Multispectral remote sensing information was obtained by multispectral sensors for 11 bands at $100 \mathrm{~m}$ altitude. Parameters such as RF, LOG (1/RF), RVI and NDVI were extremely correlated with the SPAD value. Both the univariate and multiple linear regression prediction models showed good predictive effects. Both the univariate linear regression models, such as $\mathrm{RVI}_{(570 / 800)}$ and $\operatorname{NDVI}_{(570,800)}$, and the multiple linear regression models, such as $\operatorname{RVI}_{(570 / 800,570 / 950,700 / 840,700 / 900)}$ and $\operatorname{NDVI}_{[(570,800),(570,950),(700,840)] \text {, }}$ showed the best predictive effect for the SPAD value in this work.

The spatial distribution maps of chlorophyll content were 
formed by using $\mathrm{NDVI}_{[(570,800),(570,950),(700,840)]}$, which can directly reflect the chlorophyll content level and its spatial distribution in the orchard. The establishment and improvement of this technical system should provide a reference point and evidence for precise nutrition management of citrus orchards.

\section{Acknowledgements}

The authors acknowledge that this work was financially supported by the China National Key Research and Development Project (2016YFD0200703), the China National Science \& Technology Support Program (2014BAD16B0103), the China Chongqing Science \& Technology Support \& Demonstration Project (CSTC2014fazktpt80015), and the Jiangxi Province 2011 Collaborative Innovation Special Funds "Co-Innovation Center of the South China Mountain Orchard Intelligent Management Technology and Equipment" (Jiangxi Finance Refers to [2014] No. 156).

\section{[References]}

[1] Smith P F, Reuther W. Citrus nutrition. in Temperate to Tropical Fruit Nutrition, Horticultural Publications. Rutgers-The State University, New Jersey, 1966; pp.174-207.

[2] Nijs I, Behaeghe T, Impens I. Leaf nitrogen content as a predictor of photosynthetic capacity in ambient and global change conditions. Journal of Biogeography, 1995; 177-183.

[3] Wood C W, Reeves D W, Himelrick D G. Relationships between chlorophyll meter readings and leaf chlorophyll concentration, $\mathrm{N}$ status, and crop yield: A review. Proc. Agron. Soc. NZ, 1993; 23: 1-9.

[4] Guan J Y, Hao Z B, Zhang D, Wang X L. Detection and biological function of chlorophyll. Journal of Northeast Agricultural University, 2009; 12: 130-134. (in Chinese)

[5] Cerovic Z G, Masdoumier G, Ghozlen N B, Latouche G. A new optical leaf clip meter for simultaneous non-destructive assessment of leaf chlorophyll and epidermal flavonoids. Physiologia Plantarum, 2012; 146(3): 251-260.

[6] Netto A T, Campostrini E, de Oliveira J G, Bressan-Smith R E. Photosynthetic pigments, nitrogen, chlorophyll a fluorescence and SPAD-502 readings in coffee leaves. Scientia Horticulturae, 2005; 104(2): 199-209.

[7] Hoel B O, Solhaug K A. Effect of irradiance on chlorophyll estimation with the Minolta SPAD-502 leaf chlorophyll meter. Annals of Botany, 1998; 82(3): 389-392.

[8] Markwell J, Osterman J C, Mitchell J L. Calibration of the Minolta SPAD-502 leaf chlorophyll meter. Photosynthesis Research, 1995; 46(3): $467-472$.

[9] Li G H, Xue L H, You J, Wang S H. Spatial distribution of leaf N content and SPAD value and determination of the suitable leaf for $\mathrm{N}$ diagnosis in rice. Scientia Agricultura Sinica, 2007; 40(6): 1127-1134.

[10] Asai H, Samson B K, Stephan H M, Songyikhangsuthor K, Homma K, Kiyono $\mathrm{Y}$, et al. Biochar amendment techniques for upland rice production in Northern Laos: 1. Soil physical properties, leaf SPAD and grain yield. Field Crops Research, 2009; 111(1): 81-84.

[11] Pinar A, Curran P J. Technical note grass chlorophyll and the reflectance red edge. International Journal of Remote Sensing, 1996; 17(2): 351-357.
[12] Gitelson A A, Merzlyak M N, Lichtenthaler H K. Detection of red edge position and chlorophyll content by reflectance measurements near $700 \mathrm{~nm}$. Journal of Plant Physiology, 1996; 148(3): 501-508.

[13] Wang X, Ji H Y. Quantitative analysis of chlorophyll in wheat leaf based on reflection spectroscopy and transmission spectroscopy using portable spectrometer. Chinese Agricultural Science Bulletin, 2011; 21: 39-43. (in Chinese)

[14] Zhang H, Yao X G, Zhang X B, Zhu L L, Ye S T, Zheng K F, et al. Measurement of rice leaf chlorophyll and seed nitrogen contents by using Multi-Spectral imagine. Chinese Journal of Rice Science, 2008; 5: 555-558. (in Chinese)

[15] Deng X L, Li M Z, Zheng L H, Zhang Y, Sun H. Estimating chlorophyll content of apple leaves based on preprocessing of reflectance spectra. Transactions of the CSAE, 2014; 14: 140-147. (in Chinese)

[16] Yue X J, Quan D P, Hong T S, Wang J, Qu X M, Gan H M. Non-destructive hyperspectral measurement model of chlorophyll content for citrus leaves. Transactions of the CSAE, 2015; 1: 294-302. (in Chinese)

[17] Song X Y, Wang J H, Yang G J, Gui B, Chang H. Winter wheat GPC estimation based on leaf and canopy chlorophyll parameters. Spectroscopy and Spectral Analysis, 2014; 7: 044. (in Chinese)

[18] Wang Y J. Analysis of correlation coefficient and coefficient of determination. Academic Annual Conference of Science and Technology Journals in the Yangtze River Basin and the Northwest Region of China, 2008.

[19] Fang X Y, Zhu X C, Wang L, Zhao G X. Hyperspectral monitoring of the canopy chlorophyll content at apple tree prosperous fruit stage. Scientia Agricultura Sinica, 2013; 16: 3504-3513.

[20] Geladi P, Kowalski B R. Partial least-squares regression: A tutorial Analytica Chimica Acta, 1986; 185: 1-17.

[21] Wang Z P, Zhou G H, Luo G G. Partial least square method (PLS) and its application in analytical chemistry. Analytical Chemistry, 1989; 17(7): 662-669.

[22] Wang Q, Yi Q X, Bao A M, Luo Y, Zhao J. Estimating chlorophyll density of cotton canopy by hyperspectral reflectance. Transactions of the CSAE, 2012; 15:125-132. (in Chinese)

[23] Galvao R K H, Araujo M C U, Jose G E, Pontes M J C, Silva E C, Saldanha T C B. A method for calibration and validation subset partitioning. Talanta, 2005; 67(4): 736-740.

[24] Uddling J, Gelang-Alfredsson J, Piikki K, Pleijel H. Evaluating the relationship between leaf chlorophyll concentration and SPAD-502 chlorophyll meter readings. Photosynthesis Research, 2007; 91(1): 37-46.

[25] Zhang X, Yuan H F, Guo Z, Song C F, Li X Y, Xie J C. Study of the over-fitting in building PLS model using orthogonal signal correction. Spectroscopy and Spectral Analysis, 2011; 6: 1688-1691. (in Chinese)

[26] Wichern D W. Applied multivariate statistical analysis. Tsinghua University Press Co., Ltd., 2001.

[27] Huang J F, Blackburn G A. Optimizing predictive models for leaf chlorophyll concentration based on continuous wavelet analysis of hyperspectral data. International Journal of Remote Sensing, 2011; 32(24): 9375-9396.

[28] Tian X, He S L, Lyu Q, Yi S L, Xie R J, Zheng Y Q, et al. Determination of photosynthetic pigments in citrus leaves based on hyperspectral images data. Spectroscopy and Spectral Analysis, 2014; 9: 45. (in Chinese)

[29] Liu X F, Lyu Q, He S L, Yi S L, Xie R J, Zheng Y Q, et al. Estimation of nitrogen and pigments content in citrus canopy by low-altitude remote sensing. Journal of Remote Sensing, 2015; 19(6): 1007-1018. (in Chinese) 